\title{
Co-occurrence of CALR and MPL somatic mutations in an Indian patient with a Philadelphia-negative myeloproliferative neoplasm
}

\author{
Ketki Kelkar ${ }^{1,2,3}$ - Vijay Ramanan ${ }^{2,4} \cdot$ Siddharth Anand $^{3} \cdot$ Shatakshi Ranade ${ }^{3} \cdot$ Kunal Patil $^{3} \cdot$ Meenal Agarwal $^{3,5}$. \\ Nikhil Phadke ${ }^{3,5}$
}

Received: 15 March 2019 / Accepted: 20 May 2019 / Published online: 8 June 2019

(C) Springer-Verlag GmbH Germany, part of Springer Nature 2019

\begin{abstract}
Philadelphia-negative myeloproliferative neoplasms (MPNs) are a group of clonal disorders that are characterized by excessive proliferation of abnormal myeloid precursors and mature cells. Somatic driver mutations in the JAK2, CALR, and MPL genes serve as major diagnostic criteria in the classification of the MPNs, namely polycythemia vera (PV), essential thrombocythemia (ET), and primary myelofibrosis (PMF). Although initially thought to be mutually exclusive, recent studies have reported the coexistence of JAK2, MPL, and CALR mutations. In this case report, we describe a case of a Philadelphia-negative myeloproliferative neoplasm harboring mutations in the CALR [NM_004343.3:c.1092_1143del52 (NP_004334.1:p.Leu367Thrfs)] and MPL [NM_005373.2:c.1543T>A (NP_005364.1:p.Try515Ärg)] genes. Given the rarity of documented co-occurrence of driver mutations in these two genes and the concomitant paucity of data regarding management of patients harboring mutations in both these genes simultaneously, there are no clear guidelines for the treatment of patients with these mutation patterns and hence it is difficult to assess the true relevance of this genotype. The fact that both the MPL and CALR genes are a part of the JAK-STAT pathway could, however, assist in the clinical decision-making process.
\end{abstract}

Keywords $C A L R \cdot$ Doubly mutated $\cdot$ Essential thrombocythemia $\cdot M P L \cdot$ Myeloproliferative neoplasm $\cdot$ Next-generation sequencing

\section{Introduction}

The classical Philadelphia-negative myeloproliferative neoplasms (MPNs), consisting of polycythemia vera (PV), essential thrombocythemia (ET), and primary myelofibrosis (PMF), are heterogeneous clonal neoplasms with somatic driver mutations in the Janus kinase 2 (JAK2), calreticulin (CALR), and myeloproliferative leukemia $(M P L)$ virus oncogene genes [1]. Mutations in these genes were initially thought to be mutually

Ketki Kelkar

ketki.kelkar@genepathdx.com

1 Anjali Diagnostic Pathology Laboratory, Tilak Road, Pune 411002, India

2 MVR Welfare Foundation, Pune 411009, India

3 GenePath Diagnostics India Pvt Ltd, 5th Floor, Phadke Hospital, 1260/B Jangli Maharaj Road, Shivajinagar, Pune 411004, India

4 Yashoda Hematology Clinic, Tilak Road, Pune 411002, India

5 I-SHARE Foundation, J.M. Road, Pune 411004, India exclusive. Recently, however, we have encountered two cases with concurrent mutations in two of these genes. One of these was a case of myelofibrosis with concurrent mutations in $J A K 2$ exon 14 and $M P L$ exon 10; the other was a patient with concurrent mutations in the CALR and MPL genes. Multiple research groups have recently reported similar findings with co-existence of a JAK2 mutation either with a $C A L R$ or an $M P L$ gene mutation [2-9]. There are, however, limited reports of concurrent $C A L R$ and $M P L$ gene mutations [5, 10]. Here, we describe a case of Philadelphia-negative myeloproliferative neoplasm with concurrent $C A L R$ and $M P L$ mutations. To the best of our knowledge, this is the first report of a $C A L R$ and $M P L$ double mutant from India.

\section{Clinical history}

A 57-year-old-male was referred to a clinical hematologist for moderate splenomegaly in 2012. The patient had a history of high platelet count since 1994; blood work-up reports were, however, not available. The bone marrow 
procedure, performed in 1994, was reported from a lab with limited resources. Hemogram values and bone marrow aspirate microscopy findings from the 1994 procedure were, unfortunately, unavailable. The trephine biopsy report stated that the marrow was mildly hypocellular with an increase in the number of megakaryocytes, which were seen in clusters as well as singly scattered. There was no mention of megakaryocytic morphology in the biopsy report. The report also stated that myeloid or erythroid hyperplasia was not observed. Although the report mentioned mild fibrosis (verified by reticulin staining at the time), the grade was not mentioned. In view of the mild fibrosis, a diagnosis of primary myelofibrosis was unlikely. The trephine biopsy slides were, unfortunately, unavailable at the time of writing this report. Therefore, a confirmation of the 1994 findings was not possible. In 2012, molecular testing requested by the treating hematologist from a peripheral blood sample did not show the presence of the JAK2 V617F mutation, which is commonly associated with $\mathrm{Ph}$-negative myeloproliferative neoplasms. A real-time quantitative PCR (qPCR) was also performed at that time to rule out chronic myeloid leukemia (CML). $B C R-A B L 1$ fusion transcripts were detected at $0.13 \%$ compared with the reference $A B L$ transcript. No CML-specific treatment was initiated; however, the patient was initiated on aspirin. It is assumed that this course of action was taken to reduce the risk of thrombosis by the treating physician. The patient stated that he followed up with the hematologist quarterly for the next 3 years. His platelet count during this period was reported to be normal; however, no reports from this period are available. It should be noted that the patient indicated that he had self-medicated with traditional Indian herbal remedies (Ayurvedic) including Guduchi (Tinospora cordifolia, reported to be an immunity booster) and Sharpunkha (Tephrosia purpurea, reported to protect the liver and spleen and boost hemoglobin levels). The effect, if any, of this self-medication has not been determined. In 2015, the patient underwent surgery for right-sided inguinal hernia, after which he developed ascites and was started on a diuretic (torsemide (Dytor)), which alleviated the ascites. In May 2018, he was referred to our multidisciplinary team for a full work-up. In view of the moderate splenomegaly, which could not be explained solely by mild liver parenchymal disease, our hematologist requested for sequence analysis of a panel of genes associated with myeloproliferative neoplasms (MPNs). In addition, the hematologist requested a repeat testing for hemogram, liver function, and creatinine levels. The peripheral smear from the 2018 hemogram indicated a normocytic normochromic anemia (hemoglobin of $8.8 \mathrm{~g} / \mathrm{dl}$ ) with presence of tear-drop cells and target cells; no nucleated RBCs were observed. The total leucocyte counts and differential were within normal limits. Interestingly, the platelet counts were also within normal limits. Bone marrow testing was not performed at this time because the patient refused to undergo a repeat procedure. Based on current available information, a diagnosis of a Philadelphia-negative myeloproliferative neoplasm, not subclassified, was made.

\section{Materials and methods}

Testing of DNA extracted from the patient's peripheral blood was performed by paired-end custom amplicon nextgeneration sequencing (NGS) as described previously [11], with a slight modification to the protocol. Instead of a direct PCR-based incorporation of sequencing adapters and multiplexing tags, the generated amplicons (JAK2 exons 12 and 14, CALR exon 9, and MPL exon 10) were first subjected to transposon-mediated adapter incorporation (tagmentation) to improve compatibility with shorter-read NGS protocols. Multiplexing tags were incorporated subsequently using a PCR-based method. Sequencing was performed on an Illumina NextSeq 500 sequencer instrument using sequencing-by-synthesis (SBS) chemistry in $2 \times 150$ pairedend mode. Bioinformatic analysis was performed using an inhouse custom-developed pipeline.

\section{Results}

A total of 34,606 sequence reads were obtained for this sample. More than $90 \%$ of these sequences had a $Q$-score $>30$. No pathogenic or clinically relevant somatic variants were detected in exons 12 and 14 of the JAK2 gene. However, two known somatic mutations were detected at clinically relevant allelic depths in exon 9 of the CALR gene and exon 10 of the $M P L$ gene.

A classical "type-1" 52-bp CALR deletion, [ $\mathrm{NM}_{-} 004343.3$ : c. 1092 _ $1143 \mathrm{~d}$ e 152 (NP_004334.1:p.Leu367Thrfs)], was detected at a mutant allele frequency of approximately $13 \%$ with a coverage of more than 4500 reads (Fig. 1). A mutation in the MPL gene, [NM_005373.2:c.1543T>A (NP_005364.1:p.Try515Arg)], was detected at approximately $9.8 \%$ allelic depth with a total coverage of greater than 3300 reads (Fig. 2). Alignment screenshots are from Golden Helix GenomeBrowse ${ }^{\circledR}$ visualization tool v2.x by Golden Helix, Inc. [12].

\section{Discussion}

The 2016 WHO classification of myeloproliferative neoplasms states that approximately $80 \%$ patients with ET harbor 


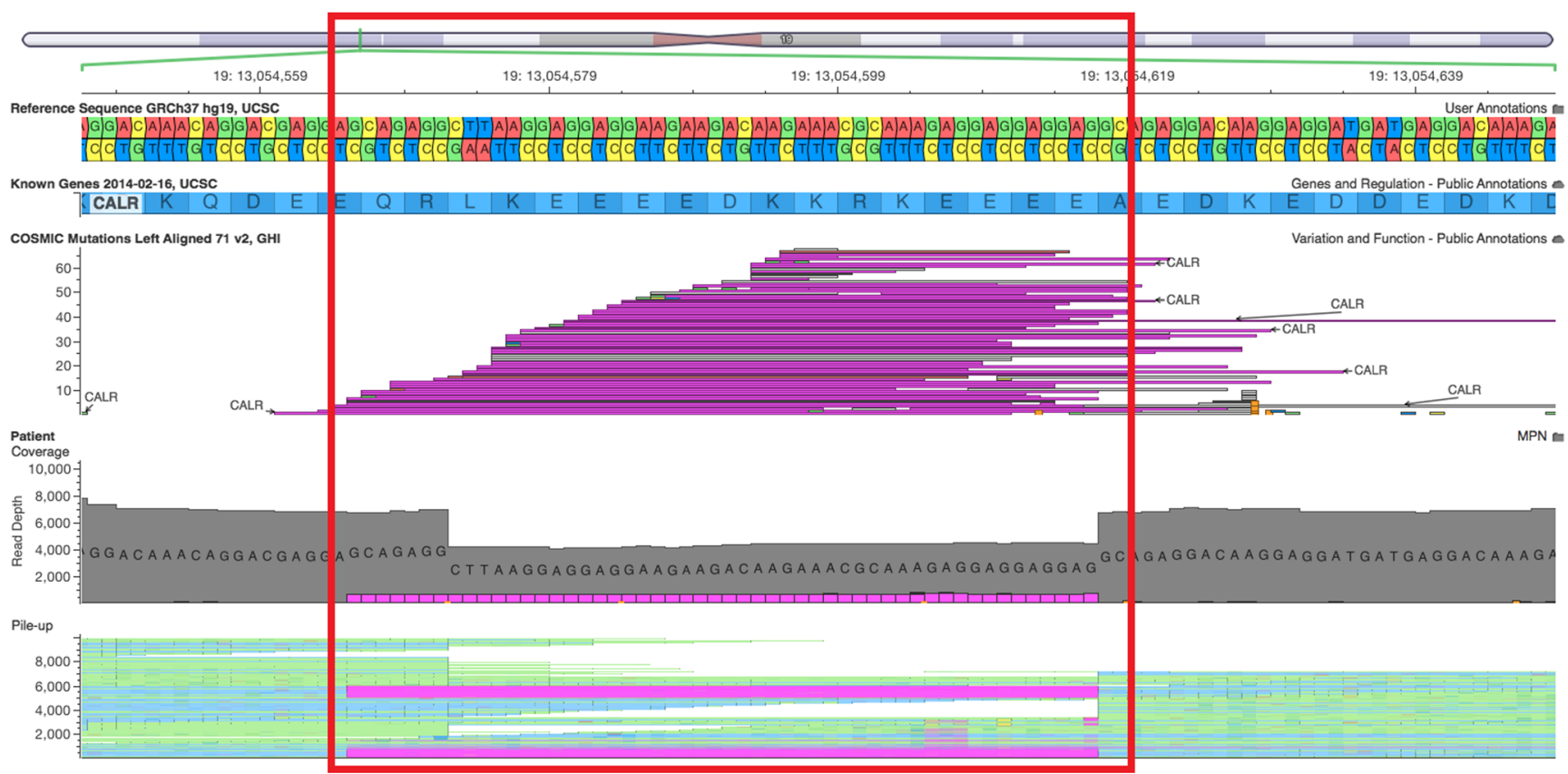

Fig. 1 Alignment of reads for exon 9 of the $C A L R$ gene. The figure shows alignment of the reads obtained from the patient's DNA for exon 9 of the $C A L R$ gene against the reference genome and the COSMIC database. The region of interest is highlighted by a red box. Deletions in the patient's reads (last two tracks) are highlighted in pink

mutations in the JAK2, CALR, and MPL genes were initially thought to be mutually exclusive; however, there have been recent reports of "doubly mutated" (DM) patients. We have summarized previously published literature of DM cases in Table 1. The types of mutations have been mentioned in the table only in instances where they have been mentioned in the corresponding study.

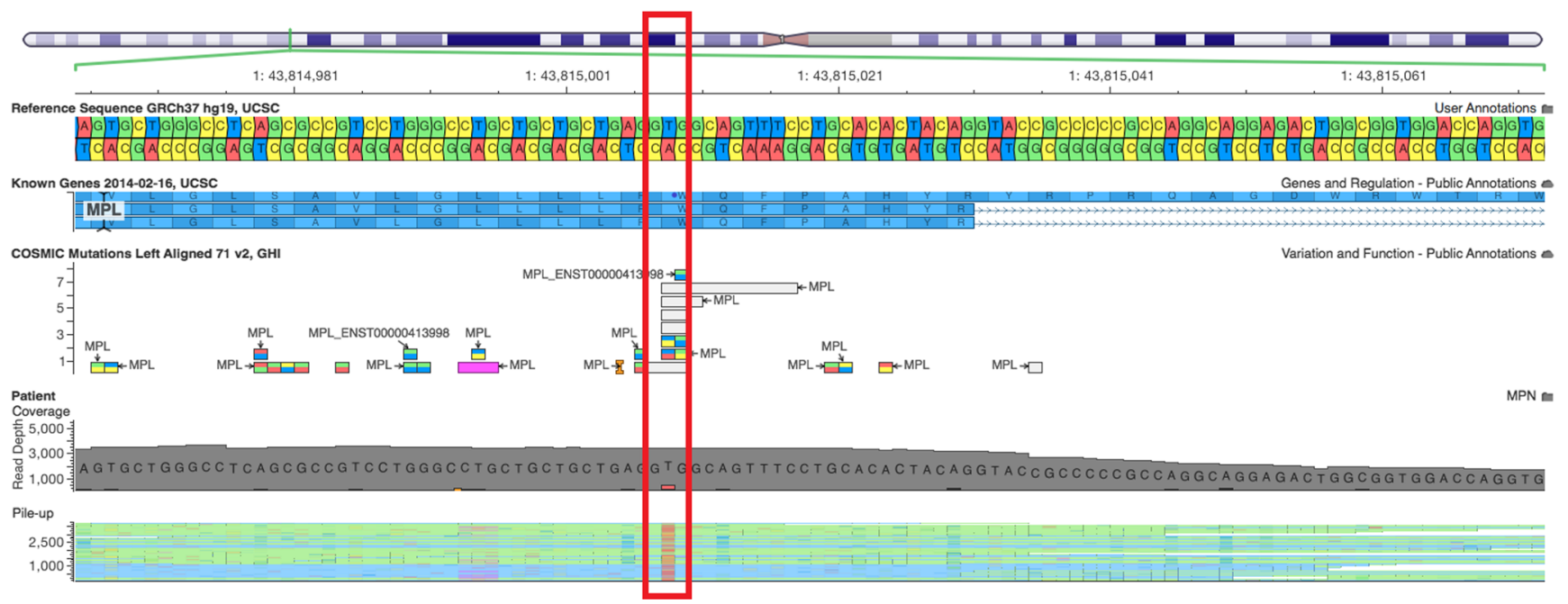

Fig. 2 Alignment of reads for exon 10 of the $M P L$ gene. The figure shows alignment of the reads obtained from the patient's DNA for exon 10 of the MPL gene against the reference genome and the COSMIC database. The region of interest is highlighted by a red box. The mutation is represented in red in the patient's reads (last two tracks) 
Table 1 Studies in which concomitant $J A K 2, M P L$, and $C A L R$ mutations have been observed

\begin{tabular}{|c|c|c|c|c|c|c|c|}
\hline \multirow[t]{2}{*}{ Authors } & \multirow{2}{*}{$\begin{array}{l}\text { Year of } \\
\text { publication }\end{array}$} & \multirow{2}{*}{$\begin{array}{l}\text { Number } \\
\text { of patients }\end{array}$} & \multirow{2}{*}{$\begin{array}{l}\text { MPN } \\
\text { type }\end{array}$} & \multicolumn{4}{|l|}{ Type of mutation } \\
\hline & & & & $J A K 2$ exon 14 & $J A K 2$ exon 12 & $M P L$ & $C A L R$ \\
\hline McGaffin et al. [2] & 2014 & 1 & - & V617F & - & - & Type- 6 mutation (48-bp deletion) \\
\hline Kang et al. [3] & 2016 & 7 & ET & V617F & - & - & All types (types 1, 2, and 3) \\
\hline Rashid et al. [4] & 2016 & 1 & $\mathrm{ET}$ & V617F & - & - & Type-1 mutation (52-bp deletion) \\
\hline Cleyrat et al. [5] & 2017 & 1 & ET & - & - & p.W515R & Type-1 mutation (52-bp deletion) \\
\hline \multirow[t]{3}{*}{ Jeromin et al. [6] } & 2017 & 12 & - & V617F & - & Type not mentioned & - \\
\hline & & 6 & - & V617F & - & - & Type not mentioned \\
\hline & & 1 & - & - & - & Type not mentioned & Type not mentioned \\
\hline \multirow[t]{2}{*}{ Usseglio et al. [7] } & 2017 & 4 & ET & V617F & - & W515R and W515R & - \\
\hline & & 4 & ET & V617F & - & - & Type-1 mutation (52-bp deletion) \\
\hline \multirow[t]{3}{*}{ Boddu et al. [8] } & 2018 & 1 & PV & V617F (VAF 95\%) & - & $\begin{array}{l}\text { p.Y591D (VAF } \\
<10 \%)\end{array}$ & - \\
\hline & & 1 & PV & V617F (VAF 12\%) & - & p.W515L (VAF < 3\%) & - \\
\hline & & 1 & ET & V617F $(\mathrm{VAF}<1 \%)$ & - & - & Type-1 mutation (VAF 30\%) \\
\hline \multirow[t]{2}{*}{ De Roeck et al. [9] } & 2018 & 1 & $\mathrm{ET}$ & V617F & - & p.W515R & - \\
\hline & & 1 & PMF & V617F & - & - & Type-1 mutation (52-bp deletion) \\
\hline \multirow[t]{6}{*}{ Mansier et al. [10] } & 2018 & 5 & - & V617F $(\mathrm{VAF}<5 \%)$ & - & - & Type not mentioned \\
\hline & & 32 & - & V617F & - & - & Type not mentioned \\
\hline & & 11 & - & V617F & - & Type not mentioned & - \\
\hline & & 2 & - & - & - & Type not mentioned & Type not mentioned \\
\hline & & 1 & PV & V617F & $\begin{array}{l}\text { Type not } \\
\text { mentioned }\end{array}$ & - & - \\
\hline & & 1 & - & - & - & - & 2 different mutations \\
\hline This study & 2019 & 1 & - & - & - & p.W515R (VAF 9.8\%) & Type-1 52-bp deletion (VAF 13\%) \\
\hline
\end{tabular}

$V A F$ variant allele frequency

Mansier et al. [10] have previously reported that second concurrent MPN-associated mutations are more frequently encountered in patients with somatic JAK2 V617F mutant allele frequencies (MAF) of $<5 \%$. Owing to the limited number of reports describing patients with concurrent MPN mutations, it was unclear to the group whether the co-occurrence of these multiple driver mutations could affect the presentation or evolution of the disease. In a separate study, Boddu et al. [8], however, reported that second MPN-associated mutations with mutant allele frequencies of $<5 \%$ could be seen in the background in cases where the allele frequency of the primary $M P N$ mutation (JAK2 exon $14 \mathrm{~V} 617 \mathrm{~F}$ ) varied widely from 1 to $90 \%$. They surmised that the minor driver mutation could be ascribed either to low-level somatic mutations or to false positivity. Therefore, the authors suggested that a more comprehensive molecular analysis would be needed to determine whether the minor mutation was a sub-clone of the primary mutation. This could perhaps be answered by performing single-cell sequencing. Kang et al. performed a study on 123 MPN patients; seven patients with ET harbored mutations in both the JAK2 and CALR genes. They reported that co- existence of JAK2 and CALR mutations did not appear to affect the prognosis or clinical features. The authors, therefore, concluded that JAK2 mutations have a greater effect on the disease phenotype and the clinical features in ET patients than CALR mutations do. Although not directly applicable to the case at hand, the following observation from their study is of interest. They noted that ET patients harboring mutations in the JAK2 gene showed inferior progression-free survival (PFS) regardless of mutations in the CALR gene. Nevertheless, patients with mutations in the JAK2 gene exhibited an earlier response to treatment as compared with patients with mutations in the $C A L R$ gene. Based on their work published in 2014, McGaffin et al., however, were of the opinion that double-mutant patients can have a different clinical course as compared with those harboring mutations in only one of the genes.

For our patient, high-depth NGS revealed the presence of concurrent mutations in the CALR and MPL genes. A classical "type-1" CALR mutation, [NM_004343.3:c.1092_1143del52 (NP_004334.1:p.Leu367Thrfs)], was detected in exon 9 at a relative mutant allele frequency of approximately $13 \%$. An 
MPL gene mutation, [NM_005373.2:c.1543T >A (NP 005364.1:p.Try515Arg)], was also detected in exon 10 at a relative mutant allele frequency of approximately $9.8 \%$. It should be noted that testing for $B C R-A B L 1$ fusion transcripts to clarify the reported detection of Philadelphia transcripts at $0.13 \%$ at the time of initial diagnosis was also requested; however, the patient was lost to follow-up and repeat testing could not be performed. Such a low relative abundance of the $B C R-A B L 1$ transcript at the time of diagnosis is not usually considered indicative of $\mathrm{Ph}$-positive CML. Several studies have reported the presence of low levels of $B C R-A B L 1$ fusion transcripts in cases with ET and even in healthy individuals [8]. Furthermore, sequential follow-ups of a few of these patients showed disappearance of $B C R-A B L 1$ transcripts, confirming that these cases represented ET. Thus, the original detection of low levels of $B C R-A B L 1$ fusion transcript in our patient is unlikely to have been of clinical significance.

With regard to the case being discussed, the "type-1" 52-bp deletion [NM_004343.3:c.1092_1143del52 (NP_004334.1:p.Leu367Thrfs)] in the CALR gene results in a mutant protein with a novel C-terminus in which the negatively charged amino acids are replaced by neutral and positively charged amino acids, and in which the "KDEL" endoplasmic reticulum retention motif is lost. It has been suggested that impaired $\mathrm{Ca}^{2+}$ binding activity, which is known to regulate human megakaryocyte function, may play a role in mutant $C A L R$ pathogenicity [15]. Mutations in codon 515 of the MPL gene (W515L/K mutations are the most common ones in patients with ET or PMF; W515S, W515A, W515R, and $\mathrm{W} 515 \mathrm{G}$ are less frequently encountered) activate the TpoR/JAK2/STAT5 transcriptional cascade, in the absence of a ligand, leading to cellular proliferation [16]. Given that the CALR mutation in our patient was present at a mutant allele frequency of approximately $13 \%$ and the $M P L$ mutation was present at a mutant allele frequency of approximately 9.8\%, two possibilities exist: (i) the CALR mutation and the $M P L$ mutation are two independent driver mutations in distinct clonal populations, or, (ii) the $M P L$ mutation is a secondary mutation in a sub-clonal population of the $C A L R$ mutation-containing clonal population. Single-cell sequencing techniques can delineate between these two possibilities. This could help the treating clinicians in deciding whether single- or multiple-drug therapy is required for patient management.

Although rare cases of concurrent mutations in the CALR and $M P L$ genes have been reported in literature [5, 10], concurrent mutations in the CALR and MPL genes have not been reported in Philadelphia-negative myeloproliferative neoplasms in India. Of the 125 patients who have undergone concomitant testing for mutations in the JAK2, CALR, and $M P L$ genes at our center, this is the first case of a patient harboring both $C A L R$ and $M P L$ mutations. To summarize, testing for mutations in both the $C A L R$ and $M P L$ genes should be performed in the presence of low JAK2 mutation burden. Ideally, a high-depth NGS MPN panel (multiplex panel for $J A K 2, M P L$, and $C A L R$ ) capable of detecting low mutant allele burden should be performed in patients suspected of having a Philadelphia-negative chronic myeloproliferative neoplasm. There is, however, limited data regarding the clinical outcomes of patients harboring both these mutations simultaneously.

Acknowledgments We would like to thank the team from GenePath Diagnostics: Asawari Pilankar, Ashwini Bapat, Karthik Ganesan, Kavita Khatod, Neher Parimoo, Priyanka Gangodkar, Purvi Majethia, Trupti Ragte-Wathare, and Vidula Sengaonkar.

\section{Compliance with ethical standards}

Conflict of interest The authors declare that they have no conflict of interest.

\section{References}

1. Frawley T, O'Brien C, Conneally E et al (2018) Development of a targeted next-generation sequencing assay to detect diagnostically relevant mutations of JAK2, CALR, and MPL in myeloproliferative neoplasms. Genet Test Mol Biomarkers 22:98-103

2. McGaffin G, harper K, Stirling D et al (2014) JAK2 V617F and CALR mutations are not mutually exclusive; findings from retrospective analysis of a small patient cohort. Br J Hematol 167:276278

3. Kang MG, Choi HW, Lee JH et al (2016) Coexistence of JAK2 and CALR mutations and their clinical implications in patients with essential thrombocythemia. Oncotarget 7:57036-57049

4. Rashid M, Ahmed Z, Ahmed S et al (2016) Coexisting JAK2V617F and CALR exon 9 mutation in essential thrombocythaemia. Indian J Hematol Blood Transfus 32:112-116

5. Cleyrat C, Chabot-Richards DS, Lynch DT et al (2017) Leukemic transformation of post-essential thrombocythemia myelofibrosis: a unique case presenting with double MPL and CALR mutations. Blood 130:4215

6. Jeromin S, Meggendorfer M, Fasan A et al (2017) Frequency of concurrent BCR-ABL1, JAK2, CALR and MPL mutations in a cohort of 5,545 cases with suspected MPN by a deep sequencing approach. EHA Learn Center 2017:181088

7. Usseglio F, Beaufils N, Calleja A et al (2017) Detection of CALR and MPL mutations in low allelic burden JAK2 V617F essential thrombocythaemia. J Mol Diagn 91:92-98

8. Boddu P, Chihara D, Masarova L, Pemmaraju N, Patel KP, Verstovsek S (2018) The co-occurrence of driver mutations in chronic myeloproliferative neoplasms. Ann Hematol 97:20712080

9. De Roeck L, Michaux L, Debackere K et al (2018) Coexisting driver mutations in MPN: clinical and molecular characteristics of a series of 11 patients. Hematology 23:785-792

10. Mansier O, Paz DL, Lanotto JC et al (2018) Clinical and biological characterization of MPN patients harboring two driver mutations, a French intergroup of myeloproliferative neoplasms (FIM) study. Am J Hematol 93:E84-E109

11. Ramanan V, Kelkar K, Ranade S et al (2016) The clinical utility of a custom-developed targeted next-generation sequencing assay for detection of mutations associated with Philadelphia negative 
chronic myeloproliferative neoplasms: two case examples with CALR exon 9 mutations. Int J MolImmunoOncol 1:28-34

12. Bozeman, MT. Golden Helix GenomeBrowse ${ }^{\circledR}$ visualization tool (version 2.X) [software]. Golden Helix, Inc. Available from http:// www.goldenhelix.com

13. Barbui T, Thiele J, Gisslinger H, Kvasnicka HM, Vannucchi AM, Guglielmelli P, Orazi A, Tefferi A (2018) The 2016 WHO classification and diagnostic criteria for myeloproliferative neoplasms: document summary and in-depth discussion. Blood Cancer J 8:15

14. Tefferi A, Wassie EA, Guglielmelli P, Gangat N, Belachew AA, Lasho TL, Finke C, Ketterling RP, Hanson CA, Pardanani A, Wolanskyj AP, Maffioli M, Casalone R, Pacilli A, Vannucchi AM, Passamonti F (2014) Type 1 versus type 2 calreticulin mutations in essential thrombocythemia: a collaborative study of 1027 patients. Am J Hematol 89:E121-E124

15. Pietra D, Rumi E, Ferretti VV, Buduo CAD, Milanesi C, Cavalloni C, Sant'Antonio E, Abbonante V, Moccia F, Casetti IC, Bellini M,
Renna MC, Roncoroni E, Fugazza E, Astori C, Boveri E, Rosti V, Barosi G, Balduini A, Cazzola M (2016) Differential clinical effects of different mutation subtypes in $C A L R$-mutant myeloproliferative neoplasms. Leukemia 30:431-438

16. Defour JP, Chachoua I, Pecquet C, Constantinescu SN (2016) Oncogenic activation of MPL/thrombopoietin receptor by $17 \mathrm{mu}-$ tations at W515: implications for myeloproliferative neoplasms. Leukemia 30:1214-1216

Publisher's note Springer Nature remains neutral with regard to jurisdictional claims in published maps and institutional affiliations. 